\title{
ON A GEOMETRIC CONDITION RELATED TO BOUNDEDLY COMPLETE BASES AND NORMAL STRUCTURE IN BANACH SPACES
}

\author{
P. CASAZZA
}

\begin{abstract}
A basis $\left\{x_{n}\right\}$ of a Banach space $X$ is said to satisfy property $\mathrm{A}$ if for every number $C>0$ there exists a number $r_{c}>0$ such that $\left\|\sum_{i=1}^{n} \alpha_{i} x_{i}\right\|=1$ and $\left\|\sum_{i=n+1}^{\infty} \alpha_{i} x_{i}\right\| \geqq C$ imply $\left\|\sum_{i=1}^{\infty} x_{i} x_{i}\right\| \geqq 1+r_{c}$. It is known that property A implies: (1) $\left\{x_{n}\right\}$ is a boundedly complete basis of $X$, and (2) every convex, weakly compact subset of $X$ has normal structure. In this paper, we construct a reflexive Banach space $X$, with an unconditional basis $\left\{x_{n}\right\}$, such that: (a) $X$ has normal structure, and (b) there does not exist an equivalent norm on $X$ with respect to which $\left\{x_{n}\right\}$ satisfies property A; showing that the converse of (1) and (2) is invalid even with the weaker conclusion that $\left\{x_{n}\right\}$ be equivalent to a basis satisfying property $\mathrm{A}$.
\end{abstract}

1. Introduction. Let $\left\{x_{n}\right\}$ be a basis of a Banach space $X$. A. R. Lovaglia [5] defines a property for bases, which we shall call property A:

(A) For each number $C>0$, there exists a number $r_{c}>0$, such that $\left\|\sum_{i=1}^{n} \alpha_{i} x_{i}\right\|=1,\left\|\sum_{i=n+1}^{\infty} \alpha_{i} x_{i}\right\| \geqq C$ imply $\left\|\sum_{i=1}^{\infty} \alpha_{i} x_{i}\right\| \geqq 1+r_{c}$.

He then states without proof $[5$, p. 234]:

THEOREM A. If $\left\{x_{n}\right\}$ satisfies property $\mathrm{A}$, then $\left\{x_{n}\right\}$ is a boundedly complete basis.

A proof of Theorem A is given in [6] with a note that it is due to A. Pełczyński. It is then asked if every boundedly complete basis is equivalent to a basis satisfying property $\mathrm{A}$.

Property A appears again in [3], where Gossez and Lami Dozo prove

THEOREM B. If $\left\{x_{n}\right\}$ satisfies property $\mathrm{A}$, then every convex, weakly compact subset of $X$ has normal structure.

Recently, Zizler [7] has shown that every separable normed linear space can be renormed so as to have normal structure. Theorem $B$ is of particular importance as Kirk [4] proves that in a Banach space $X$, every

Received by the editors April 21, 1972

AMS (MOS) subject classifications (1969). Primary 4610; Secondary 4785.

Key words and phrases. Schauder basis, uniformly convex, locally uniformly convex, normal structure, fixed point.

(c) American Mathematical Society 1973 
nonexpansive mapping of a convex, weakly compact subset of $X$, with normal structure, into itself has a fixed point.

It is easily seen that the converse of Theorems A and B are invalid by taking a two-dimensional $l_{p}$ space and choosing two linearly independent vectors which do not satisfy property $A$. However, finite dimensional spaces can always be renormed so that a given basis satisfies property $\mathrm{A}$. We will show that the converse of Theorems A and B are invalid even with the weaker conclusion that $\left\{x_{n}\right\}$ be equivalent to a basis satisfying property A, by proving

THEOREM I. There exists a reflexive Banach space $X$, with an unconditional basis $\left\{x_{n}\right\}$, such that there does not exist an equivalent norm on $X$ with respect to which $\left\{x_{n}\right\}$ satisfies property $\mathrm{A}$.

\section{Proof of Theorem I.}

Definition 1. A Banach space is uniformly convex (u.c.) if for every $\varepsilon>0$ there exists $\delta>0$ such that whenever $\|x\|,\|y\| \leqq 1$, and $\|x-y\| \geqq \varepsilon$, then $\|(x+y) / 2\| \leqq 1-\delta$.

Definition 2. If $B_{n}$ is a Banach space with norm $\|\cdot\|_{n}$, for $n=$ $1,2,3, \cdots, P_{p}\left(\left\{B_{n}\right\}\right)$ is the Banach space of all sequences $y=\left\{y_{n}\right\}$, $y_{n} \in B_{n}$, for which $\sum_{n=1}^{\infty}\left\|y_{n}\right\|_{n}^{p}<\infty$, with the norm $\|y\|=\left(\sum_{n=1}^{\infty}\left\|y_{n}\right\|_{n}^{p}\right)^{1 / p}$.

For each natural number $n$, let $l_{n}^{\infty}$ denote $n$-dimensional Euclidean space with the sup norm $\|\cdot\|_{n}$, and let $\left\{e_{k}^{n}\right\}_{k=1}^{n}$ be the unit vector basis of $l_{n}^{\infty}$. Let $1<q<\infty$ and $X=P_{q}\left(\left\{l_{n}^{\infty}\right\}\right)$. Let $\left\{x_{n}\right\}$ be the basis of $X$ defined by:

$$
\begin{aligned}
& x_{1}=\left(e_{1}^{1},(0,0),(0,0,0), \cdots\right), \\
& x_{2}=\left(0,\left(e_{2}^{1}, 0\right),(0,0,0), \cdots\right), \\
& x_{3}=\left(0,\left(0, e_{2}^{2}\right),(0,0,0), \cdots\right),
\end{aligned}
$$

It is well known that $\left\{x_{n}\right\}$ is an unconditional basis of $X$, and that $\left\{x_{n}\right\}$ is boundedly complete (since $X$ is reflexive).

Assume there exists an equivalent norm $|\cdot|$ on $X$ with respect to which $\left\{x_{n}\right\}$ satisfies property $\mathrm{A}$. Then there exist constants $K_{1}, K_{2}>0$ such that $K_{1}|x| \leqq\|x\| \leqq K_{2}|x|$ for all $x \in X$. Let $C=K_{1} / K_{2}$ and $r_{c}$ the number defined by property A. Fix $i \geqq 1$ and let $l=2^{2 i}$. Set $p=\sum_{j=1}^{22 i} j$, and, for any $1 \leqq n \leqq 2^{2 i}-1$, let $r_{n}=\left|\sum_{k=n+1}^{p+n} x_{k}\right|$. Finally, define a sequence of scalars, $\left\{\alpha_{k}^{n}\right\}$,

$$
\begin{aligned}
\alpha_{k}^{n} & =0 & & \text { if } 1 \leqq k \leqq p \\
& =1 / r_{n} & & \text { if } p+1 \leqq k \leqq p+2 n \\
& =0 & & \text { if } p+2 n<k
\end{aligned}
$$


Then,

$$
\left|\sum_{k=1}^{p+n} \alpha_{k}^{n} x_{k}\right|=\frac{1}{r_{n}}\left|\sum_{k=p+1}^{n+n} x_{k}\right|=1
$$

and,

$$
\begin{aligned}
r_{n}=\left|\sum_{k=p+1}^{n+n} x_{k}\right| & \leqq \frac{1}{K_{1}}\left\|\sum_{k=p+1}^{n+n} x_{k}\right\| \\
& =\frac{1}{K_{1}}\left\|\sum_{k=1}^{n} e_{k}^{l}\right\|_{l}=\frac{1}{K_{1}} \quad \text { for } n=1,2, \cdots .
\end{aligned}
$$

Therefore,

$$
\begin{aligned}
\left|\sum_{k=n+n+1}^{\infty} \alpha_{k}^{n} x_{k}\right| & =\frac{1}{r_{n}}\left|\sum_{k=p+n+1}^{p+2 n} x_{k}\right| \\
& \geqq \frac{1}{K_{2}} \frac{1}{r_{n}}\left\|\sum_{k=p+n+1}^{p+2 n} x_{k}\right\| \\
& \geqq \frac{K_{1}}{K_{2}}\left\|\sum_{k=n+1}^{2 n} e_{k}^{l}\right\|_{l}=\frac{K_{1}}{K_{2}}=C .
\end{aligned}
$$

Then, since $\left\{x_{n}\right\}$ satisfies property A,

$$
\left|\sum_{k=1}^{\infty} \alpha_{k}^{n} x_{k}\right|=\frac{1}{r_{n}}\left|\sum_{k=p+1}^{p+2 n} x_{k}\right| \geqq 1+r_{c},
$$

and by (2.1), for all $1 \leqq n \leqq 2^{2 i-1}$, we have,

$$
\frac{\left|\sum_{k=p+1}^{p+2 n} x_{k}\right|}{\left|\sum_{k=p+1}^{p+n} x_{k}\right|}=\frac{\left(1 / r_{n}\right)\left|\sum_{\substack{k+2 n \\ k=p+1}}^{p+2} x_{k}\right|}{\left(1 / r_{n}\right)\left|\sum_{\substack{p+n \\ k=p+1}}^{p+n} x_{k}\right|}=\frac{1}{r_{n}}\left|\sum_{\substack{p+2 n \\ k=p+1}} x_{k}\right| \geqq 1+r_{c} .
$$

Applying (2.2),

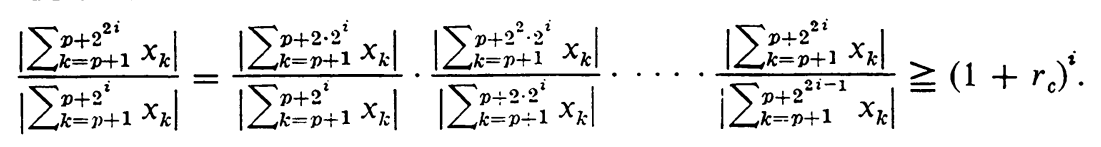

Also,

$$
\frac{\left|\sum_{\substack{p+2^{2 i} \\ k=1}} x_{k}\right|}{\left|\sum_{\substack{p+2^{i} \\ k=p+1}} x_{k}\right|} \leqq \frac{\left(1 / K_{1}\right)\left\|\sum_{k=p+1}^{p+2^{2 i}} x_{k}\right\|}{\left(1 / K_{2}\right)\left\|\sum_{\substack{p+2^{i} \\ k=p+1}}^{p} x_{k}\right\|}=\frac{K_{2}}{K_{1}} \frac{\left\|\sum_{k=1}^{2^{2 i}} e_{k}^{l}\right\|_{l}}{\left\|\sum_{k=1}^{2^{i}} e_{k}^{l}\right\|_{l}}=\frac{K_{2}}{K_{1}}
$$

However, for sufficiently large $i,\left(1+r_{c}\right)^{i}>K_{2} / K_{1}$. This contradiction concludes the proof of Theorem I.

Using the result of Zizler, we obtain a Banach space with normal structure satisfying Theorem I. However, it was pointed out by the referee that the following procedure will yield concrete examples of Banach 
spaces which are locally uniformly convex, have normal structure, and satisfy Theorem I. Let $p_{n}$ be such that $p_{n}<\infty$ and $l_{p_{n}}^{n}$ is $\frac{1}{2}$-isometric to $l_{n}^{\infty}$. Then $P_{2}\left(\left\{l_{p_{n}}^{n}\right\}\right)$ has the desired properties by [1], [5], and the argument given above. By applying our technique to the space $d$ of Sargent (see [6, p. 361]) we see that the unit vector basis of $d$ is an unconditional, symmetric, boundedly complete, and nonshrinking basis which is not equivalent to any basis satisfying property A. Finally, we note that it follows from Day [2, p. 315] that none of the Banach spaces discussed above is isomorphic to a uniformly convex space.

3. Uniformly convex spaces. Singer [6, Theorem 6.2, p. 288] shows that property $\mathrm{A}$ is equivalent to:

(B) For every $\varepsilon>0$ there exists a $\delta>0$, such that $\left\|\sum_{i=1}^{n} \alpha_{i} x_{i}\right\|>1-\delta$, $\left\|\sum_{i=1}^{\infty} \alpha_{i} x_{i}\right\|=1$ imply $\left\|\sum_{i=n+1}^{\infty} \alpha_{i} x_{i}\right\| \leqq \varepsilon$.

It is clear from the two-dimensional example described earlier that if a Banach space $X$ is u.c., then a basis of $X$ need not satisfy property A. However, it is interesting that we do have:

THEOREM II. If $\left\{x_{n}\right\}$ is a monotone basis of a u.c. space $X$, then $\left\{x_{n}\right\}$ satisfies property $\mathrm{B}$ (hence, property $\mathrm{A})$.

Proof. Given $\varepsilon>0$, choose $\delta>0$ satisfying Definition 1. Assume there exists a sequence of scalars $\left\{\alpha_{n}\right\}$ such that

$$
\left\|\sum_{i=1}^{n} \alpha_{i} x_{i}\right\|>1-\delta, \quad\left\|\sum_{i=1}^{\infty} \alpha_{i} x_{i}\right\|=1, \quad \text { and }\left\|\sum_{i=n+1}^{\infty} \alpha_{i} x_{i}\right\|>\varepsilon .
$$

Then, $\left\{x_{n}\right\}$ is monotone implies

$$
1-\delta<\left\|\sum_{i=1}^{n} \alpha_{i} x_{i}\right\| \leqq\left\|\sum_{i=1}^{\infty} \alpha_{i} x_{i}\right\|=1
$$

If $x=\sum_{i=1}^{n} \alpha_{i} x_{i}$ and $y=\sum_{i=1}^{\infty} \alpha_{i} x_{i}$, then $\|x\|,\|y\| \leqq 1$, and $\|x-y\|=$ $\left\|\sum_{i=n+1}^{\infty} \alpha_{i} x_{i}\right\|>\varepsilon$, so that $\|(x+y) / 2\| \leqq 1-\delta$. Hence,

$$
1-\delta \geqq\left\|\frac{x+y}{2}\right\|=\left\|\sum_{i=1}^{n} \alpha_{i} x_{i}+\frac{1}{2} \sum_{i=n+1}^{\infty} \alpha_{i} x_{i}\right\| \geqq\left\|\sum_{i=1}^{n} \alpha_{i} x_{i}\right\|>1-\delta,
$$

which is clearly a contradiction.

By Theorem II, it is natural to ask:

Problem I. If $\left\{x_{n}\right\}$ is a basis of a u.c. space $X$, does there exist an equivalent u.c. norm on $X$ such that $\left\{x_{n}\right\}$, with respect to the new norm, is a monotone basis? If not, does there at least exist an equivalent norm on $X$ with respect to which $\left\{x_{n}\right\}$ satisfies property A ?

I wish to express my gratitude to the referee for greatly simplifying the material contained in this paper. 


\section{REFERENCES}

1. L. P. Belluce, W. A. Kirk and E. F. Steiner, Normal structure in Banach spaces, Pacific J. Math. 26 (1968), 433-440. MR 38 \#1501.

2. M. M. Day, Reflexive Banach spaces not isomorphic to uniformly convex spaces, Bull. Amer. Math. Soc. 47 (1941), 313-317. MR 2, 221.

3. J. P. Gossez and E. Lami Dozo, Structure normal et base de Schauder, Acad. Roy. Belg. Bull. Cl. Sci. (5) 55 (1969), 673-681. MR 41 \#795.

4. W. A. Kirk, A fixed point theorem for mappings which do not increase distances, Amer. Math. Monthly 72 (1965), 1004-1006. MR 32 \#6436.

5. A. R. Lovaglia, Locally uniformly convex Banach spaces, Trans. Amer. Math. Soc. 78 (1955), 225-238. MR 16, 596.

6. Ivan Singer, Bases in Banach spaces, Springer-Verlag, New York, 1970.

7. V. Zizler, Some notes on various rotundity and smoothness properties of separable Banach spaces, Comment. Math. Univ. Carolinae 10 (1969), 195-206. MR 39 \#7401.

Department of Mathematics, University of Iowa, Iowa City, Iowa 52240

Current address: Department of Mathematics, University of Alabama in Huntsville, Huntsville, Alabama 35807 